\title{
The Importance of Pyrazinamide in Antimycobacterial Combination Therapy of BCG Disease: Do We Need a Treatment Guideline in The Future?
}

\author{
Ikhwanuliman Putera $^{1,}$; Ikrar Syahmar $^{1}$ \\ ${ }^{1}$ Faculty of Medicine, University of Indonesia, Jakarta, Indonesia \\ *Corresponding author: Ikhwanuliman Putera, Faculty of Medicine, University of Indonesia, Jakarta, Indonesia. Tel: +62-81933815103, E-mail: iwankings@gmail.com \\ Received: June 02, 2015; Accepted: June 04, 2015
}

Keywords: BCG Vaccine; Disseminated BCG Infection; Pyrazinamide

\section{Dear Editor,}

Tuberculosis (TB) remains a worldwide health problem as The World Health Organization (WHO) raised TB as a global health emergency (1). However, current preventive measures for TB still rely on Bacillus Calmette-Guérin (BCG) vaccination. The efficacy of this old vaccine has been questioned and the new TB vaccine candidates have been proposed (2). BCG vaccine is a live attenuated vaccine which is considered to be safe with less than $1 \%$ local adverse events in immunocompetent individuals (3). Adverse events following administration of it range from mild local complication to fatal disseminated BCG disease. Nuttall et al. (4) reported that $6 \%$ of children under antiretroviral therapy for human immunodeficiency virus (HIV) infection showed local adverse events following BCG vaccination, whereas only $0.3 \%$ of them developed disseminated BCG disease. Disseminated BCG disease cases were also reported in children who had congenital primary immunodeficiency (5).

A recent study evaluating the susceptibility pattern of BCG strains by Fahimzad et al. (6) gave insight to the selection and dose estimation of antimycobacterial drugs for treating systemic BCG disease. This study found that most antimycobacterial drugs tested alone were ineffective in limiting BCG growth in vitro; however, increasing the dose of pyrazinamide to a level of $50 \mu \mathrm{g} / \mathrm{mL}$ make $M y$ cobacterium bovis susceptible to other drugs. This finding was rather different with previous larger study identifying several BCG strains in which minimum inhibitory concentration (MIC) for several drugs were below the current finding. In Ritz et al. (3) study, all BCG strains were resistant to pyrazinamide alone with the dose of as high as $100 \mu \mathrm{g} /$ mL. Thus, using drug combination with higher dose of pyrazinamide as in Fahimzad et al. (6) study can be a solution for the treatment of disseminated BCG disease.

In the methodology section, Fahimzad et al. (6) stated that M. bovis was isolated from subaxillary adenitis, while axillary adenitis is not sufficient to confirm the diagnosis of disseminated BCG disease. Disseminated BCG disease is defined as finding $M$. bovis BCG in the distant part from vaccination site with clinical signs consistent with dissemination. M. Bovis can be isolated from distant lymph node, respiratory secretion, blood, bone marrow or cerebrospinal fluid (7). Antimycobacterial drugs had no proven benefit for the treatment of local adverse BCG vaccination events except for disseminated BCG disease (8). As this study purpose was to assess susceptibility pattern of $B C G$, readers should not be confused in how to diagnose disseminated BCG disease in the clinical practice.

Infants with positive HIV infection are contraindicated for BCG vaccination. As reported previously, disseminated BCG disease incidence increased significantly in infants with HIV, thus the risks outweigh the benefits of BCG vaccination (8). Meanwhile, study in Malawi found no $M$. bovis isolate in bloodstream from children with HIV infection who had BCG vaccination (9). Several vaccine and host-related factors can be the predisposing factors for adverse BCG vaccination events, including vaccine substrain, dose of vaccine, age of infants at the time of vaccination, and different characteristics of children in various countries (10). The program of prevention of mother to child transmission (PMTCT) endorsed by WHO, provides modification to delay BCG vaccination to prevent its serious adverse events. However, in countries without universal implementation of PMTCT program and high number of estimated HIV infection and TB incidence, disseminated BCG disease may be considerably high (8). Results from Fahimzad et al. (6) study gave valuable consideration for treatment regiment in that setting. Further pharmacokinetic and in vivo studies may be necessary to guide disseminated BCG disease treatment. 


\section{Authors' Contributions}

Ikhwanuliman Putera and Ikrar Syahmar drafted the manuscript and approved the final version of the manuscript.

\section{References}

1. World Health Organization. The Global Plan to Stop TB 2006-2015 WHO; 2015. Available from: http://www.stoptb.org/assets/documents/global/plan/globalplanfinal.pdf.

2. Hanekom WA. The immune response to BCG vaccination of newborns. Ann N Y Acad Sci. 2005;1062:69-78.

3. Ritz N, Tebruegge M, Connell TG, Sievers A, Robins-Browne R, Curtis N. Susceptibility of Mycobacterium bovis BCG vaccine strains to antituberculous antibiotics. Antimicrob Agents Chemother. 2009;53(1):316-8.

4. Nuttall JJ, Davies MA, Hussey GD, Eley BS. Bacillus CalmetteGuerin (BCG) vaccine-induced complications in children treated with highly active antiretroviral therapy. Int J Infect Dis. 2008;12(6):e99-105.
5. Roxo-Junior P, Silva J, Andrea M, Oliveira L, Ramalho F, Bezerra T, et al. A family history of serious complications due to BCG vaccination is a tool for the early diagnosis of severe primary immunodeficiency. Ital J Pediatr. 2013;39:54.

6. Fahimzad SA, Ghasemi M, Shiva F, Ghadiri K, Navidinia M, Kari mi A. Susceptibility Pattern of Bacille Calmette-Guerin Strains Against Pyrazinamide and Other Major Anti-Mycobacterial Drugs. Arch Pediatr. 2015;3(1):17814.

7. Hesseling AC, Rabie H, Marais BJ, Manders M, Lips M, Schaaf $\mathrm{HS}$, et al. Bacille Calmette-Guerin vaccine-induced disease in HIV-infected and HIV-uninfected children. Clin Infect Dis. 2006;42(4):548-58.

8. Hesseling AC, Johnson LF, Jaspan H, Cotton MF, Whitelaw A, Schaaf HS, et al. Disseminated bacille Calmette-Guerin disease in HIV-infected South African infants. Bull World Health Organ. 2009;87(7):505-11.

9. Archibald LK, Kazembe PN, Nwanyanwu O, Mwansambo C, Reller LB, Jarvis WR. Epidemiology of bloodstream infections in a bacille Calmette-Guerin-vaccinated pediatric population in Malawi. J Infect Dis. 2003;188(2):202-8.

10. Chan WM, Kwan YW, Leung CW. Management of Bacillus Calmette-Guérin Lymphadenitis. HKJ Paediatr. 2011;16(2):85-94. 